\title{
Spin waves and magnetic exchange interactions in $\mathrm{CaFe}_{2} \mathrm{As}_{2}$
}

\author{
Jun Zhao ${ }^{1}$, D. T. Adroja ${ }^{2}$, Dao-Xin $\mathrm{Yao}^{3}$, R. Bewley ${ }^{2}$, Shiliang Li ${ }^{1,4}$, X. F. Wang ${ }^{5}$, G. Wu ${ }^{5}$, X. H. Chen ${ }^{5}$, \\ Jiangping $\mathrm{Hu}^{3}$ and Pengcheng Dai ${ }^{1,4,6 \star}$
}

\begin{abstract}
Antiferromagnetism is relevant to high-temperature (high- $T_{c}$ ) superconductivity because copper oxide and iron arsenide superconductors arise from electron- or hole-doping of their antiferromagnetic parent compounds ${ }^{1-6}$. There are two broad classes of explanation for antiferromagnetism: in the 'local moment' picture, appropriate for the insulating copper oxides', antiferromagnetic interactions are well described by a Heisenberg Hamiltonian ${ }^{7,8}$; whereas in the 'itinerant model', suitable for metallic chromium, antiferromagnetic order arises from quasiparticle excitations of a nested Fermi surface ${ }^{9,10}$. There has been contradictory evidence regarding the microscopic origin of the antiferromagnetic order in iron arsenide materials $s^{5,6}$, with some favouring a localized picture ${ }^{11-15}$ and others supporting an itinerant point of view ${ }^{16-20}$. More importantly, there has not even been agreement about the simplest effective ground-state Hamiltonian necessary to describe the antiferromagnetic order ${ }^{21-25}$. Here, we use inelastic neutron scattering to map spin-wave excitations in $\mathrm{CaFe}_{2} \mathrm{As}_{2}$ (refs 26, 27), a parent compound of the iron arsenide family of superconductors. We find that the spin waves in the entire Brillouin zone can be described by an effective three-dimensional local-moment Heisenberg Hamiltonian, but the large in-plane anisotropy cannot. Therefore, magnetism in the parent compounds of iron arsenide superconductors is neither purely local nor purely itinerant, rather it is a complicated mix of the two.
\end{abstract}

Since the discovery of static antiferromagnetic order (with a spin structure as in Fig. 1a) in the parent compounds of iron pnictide superconductors ${ }^{5,6}$, much effort has been focused on understanding the role of spin dynamics in the superconductivity of these materials ${ }^{11-20}$. A determination of the effective magnetic exchange coupling and ground-state Hamiltonian in the parent compounds of these materials is important because such an understanding will provide the basis against which superconductivity-induced changes can be identified. Using inelastic neutron scattering, we have measured the dispersion of spin-wave excitations in $\mathrm{CaFe}_{2} \mathrm{As}_{2}$ (refs 26, 27), one of the parent compounds of the FeAsbased superconductors, and determined the effective magnetic exchange interactions. If the static long-range antiferromagnetic order shown in Fig. 1a for the parent compounds of iron-based superconductors originates from a collective spin-density-wave order instability of itinerant electrons like in chromium, the velocity of spin-wave excitations $c$ should be $c=\left(v_{\mathrm{e}} v_{\mathrm{h}} / 3\right)^{1 / 2}$, where $v_{\mathrm{e}}$ and $v_{\mathrm{h}}$ are the electron and hole Fermi velocity, respectively ${ }^{9}$. Furthermore, spin-wave excitations should exhibit longitudinal and transverse polarization, and damp into single-particle excitations (Stoner continuum) through the transfer of an electron (spin) from the majority to the minority band at high energies as shown schematically in Fig. 1c (ref. 10). On the other hand, if magnetic order in iron pnictides has a local moment origin as in the parent compounds of the copper oxides ${ }^{1}$, one should observe well-defined (essentially instrumental resolution limited) spin waves throughout the Brillouin zone and magnetic coupling between local moments should be dominated by direct and superexchange interactions (Fig. 1d) ${ }^{11-15}$. In recent neutron scattering experiments, the presence of itinerant magnetic excitations and a Stoner continuum have been suggested in $\mathrm{BaFe}_{2} \mathrm{As}_{2}$ (ref. 24) and $\mathrm{CaFe}_{2} \mathrm{As}_{2}$ (ref. 25). Whereas low-energy spin waves in $\mathrm{CaFe}_{2} \mathrm{As}_{2}$ can be described by a classical Heisenberg Hamiltonian, a Stoner line broadening was reported to develop above $100 \mathrm{meV}$ (or wave vector $\mathbf{Q}=(1.2,0,1)$ reciprocal lattice units (r.l.u.) or $\sim 0.2$ r.l.u. in reduced wave vector from the zone centre $(1,0,1))$ with no localized spin waves near the zone boundary ${ }^{25}$. Furthermore, the authors find that a Heisenberg Hamiltonian with effective in-plane nearest-neighbours (Fig. $1 \mathrm{a}, J_{1 \mathrm{a}}$ and $J_{1 \mathrm{~b}}$ ), next-nearestneighbour (Fig. 1a, $J_{2}$ ) and out-of-plane (Fig. 1a, $J_{c}$ ) exchange couplings of $S\left(J_{1 \mathrm{a}}+J_{1 \mathrm{~b}}\right)=44, S J_{2}=31 \pm 3$ and $S J_{\mathrm{c}}=4.5 \pm 1 \mathrm{meV}$ (where spin $S=1$ ) can best describe spin waves of $\mathrm{CaFe}_{2} \mathrm{As}_{2}$ below $100 \mathrm{meV}$ (ref. 25). Although these results are interesting, they are similar to earlier work ${ }^{21-24}$ and have not determined the effective ground-state Hamiltonian because the signs of the effective change coupling constants (Fig. $1 \mathrm{a}, J_{\mathrm{la}}$ and $J_{\mathrm{lb}}$ ) can be determined only by zone-boundary spin-wave data, which are lacking in ref. 25. A correct determination of all exchange coupling constants $\left(J_{1 \mathrm{a}}, J_{1 \mathrm{~b}}\right.$ and so on) is important because it enables the formation of an appropriate ground-state Hamiltonian from which superconductivity can be derived.

We used inelastic neutron scattering to study low-temperature $(T=10 \mathrm{~K})$ spin waves of single crystals of $\mathrm{CaFe}_{2} \mathrm{As}_{2}$, which has a Néel temperature of $T_{\mathrm{N}} \approx 170 \mathrm{~K}$ (refs 26,27 ). Figure 1e-1 shows two-dimensional constant-energy $(E)$ images of spin-wave excitations of $\mathrm{CaFe}_{2} \mathrm{As}_{2}$ around the antiferromagnetic zone centre in the $(H, K)$ scattering plane ${ }^{21-25}$. Previous low-energy measurements ${ }^{23}$ revealed that spin waves in $\mathrm{CaFe}_{2} \mathrm{As}_{2}$ are three-dimensional and centred at antiferromagnetic wave vector $\mathbf{Q}=(1,0, L=1,3,5, \ldots)$ r.l.u. For energy transfers of $E=48 \pm 6$ (Fig. 1e) and $65 \pm 4 \mathrm{meV}$ (Fig. 1f), spin waves are still peaked at $\mathbf{Q}=(1,0, L=1,3,5)$ r.l.u. in the centre of the Brillouin zone (shown as dashed rectangles). As the energy increases to $E=100 \pm 10$ (Fig. 1g), $115 \pm 10$

\footnotetext{
${ }^{1}$ Department of Physics and Astronomy, The University of Tennessee, Knoxville, Tennessee 37996-1200, USA, ${ }^{2}$ ISIS Facility, Rutherford Appleton Laboratory, Chilton, Didcot, OX11 0QX, UK, ${ }^{3}$ Department of Physics, Purdue University, West Lafayette, Indiana 47907, USA, ${ }^{4}$ Beijing National Laboratory for Condensed Matter Physics, Institute of Physics, Chinese Academy of Sciences, Beijing 100190, China, ${ }^{5}$ Hefei National Laboratory for Physical Sciences at Microscale and Department of Physics, University of Science and Technology of China, Hefei, Anhui 230026, China, ${ }^{6}$ Neutron Scattering Science Division, Oak Ridge National Laboratory, Oak Ridge, Tennessee 37831, USA. *e-mail: daip@ornl.gov.
} 

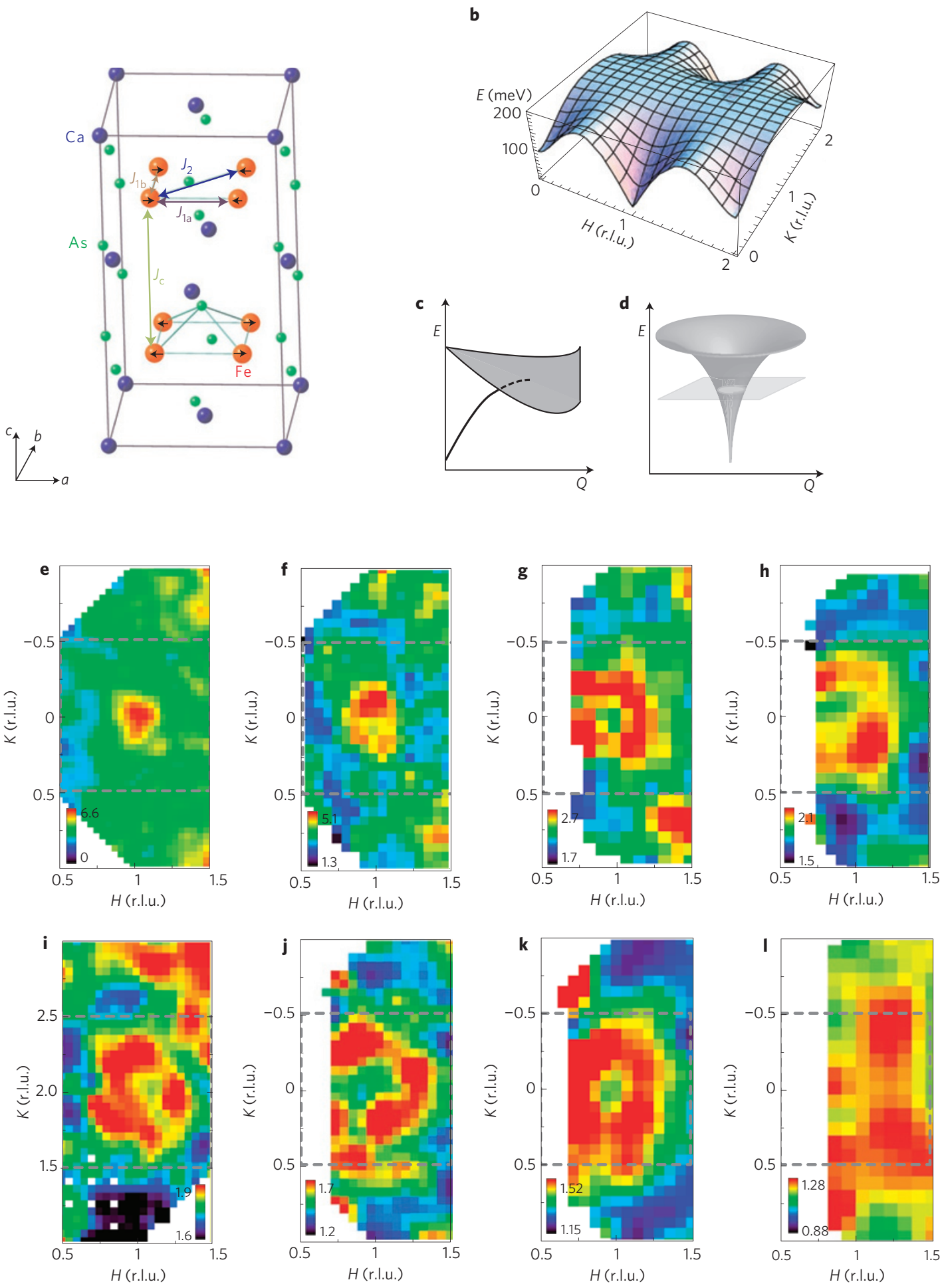

Figure 1 | Magnetic structure, calculated spin-wave dispersion and wave-vector dependence of spin-wave excitations at different energies for $\mathbf{C a F e}_{\mathbf{2}} \mathbf{A s}_{2}$. Our inelastic neutron scattering experiments were carried out on the MERLIN time-of-flight chopper spectrometer at the Rutherford Appleton Laboratory, Didcot, UK. We co-aligned $6.4 \mathrm{~g}$ of single crystals of $\mathrm{CaFe}_{2} \mathrm{As}_{2}$ grown by self-flux (with in-plane mosaic of $2^{\circ}$ and out-of-plane mosaic of $3^{\circ}$ ). The incident beam energies were $E_{\mathrm{i}}=50,80,150,200,250,450,600 \mathrm{meV}$, and mostly with $E_{\mathrm{i}}$ parallel to the $c$ axis. Spin-wave intensities were normalized to absolute units using a vanadium standard (with $30 \%$ error). We define the wave vector $\mathbf{Q}$ at $\left(q_{x}, q_{y}, q_{z}\right)$ as $(H, K, L)=\left(q_{x} a / 2 \pi, q_{y} b / 2 \pi, q_{z} c / 2 \pi\right)$ r.l.u., where $a=5.506, b=5.450$ and $c=11.664 \AA$ are the orthorhombic cell lattice parameters at $10 \mathrm{~K}$ (ref. 27). a, Schematic diagram of the Fe spin ordering in $\mathrm{CaFe}_{2} \mathrm{As}_{2}$. b. Calculated three-dimensional spin-wave dispersions using $\mathrm{SJ}_{1 \mathrm{a}}=49.9, \mathrm{SJ}_{1 \mathrm{~b}}=-5.7, \mathrm{SJ}_{2}=18.9$ and $S J_{\mathrm{c}}=5.3 \mathrm{meV}$. c, Schematic diagram for how spin-wave dispersion enters into the Stoner continuum. d, Dispersion of spin waves in a classical Heisenberg Hamiltonian. e-I, Wave-vector dependence of the spin waves for energy transfers of $E=48 \pm 6 \mathrm{meV}\left[E_{\mathrm{i}}=150 \mathrm{meV}\right.$ and $\left.\mathbf{Q}=(1,0,3)\right](\mathbf{e}) ; E=65 \pm 4 \mathrm{meV}\left[E_{\mathrm{i}}=250 \mathrm{meV}\right.$ and $\left.\mathbf{Q}=(1,0,3)\right]$ (f); $E=100 \pm 10 \mathrm{meV}\left[E_{\mathrm{i}}=450 \mathrm{meV}\right.$ and $\left.\mathbf{Q}=(1,0,3.5)\right](\mathbf{g}) ; E=115 \pm 10 \mathrm{meV}\left[E_{\mathrm{i}}=450 \mathrm{meV}\right.$ and $\left.\mathbf{Q}=(1,0,4)\right](\mathbf{h}) ; E=137 \pm 15 \mathrm{meV}\left[E_{\mathrm{i}}=600 \mathrm{meV}\right.$ and $\mathbf{Q}=(1,2,4)](\mathbf{i}) ; E=135 \pm 10 \mathrm{meV}\left[E_{\mathrm{i}}=450 \mathrm{meV}\right.$ and $\left.\mathbf{Q}=(1,0,4.5)\right](\mathbf{j}) ; E=144 \pm 15 \mathrm{meV}\left[E_{\mathrm{i}}=450 \mathrm{meV}\right.$ and $\left.\mathbf{Q}=(1,0,5)\right](\mathbf{k}) ;$ $E=175 \pm 15 \mathrm{meV}\left[E_{\mathrm{i}}=600 \mathrm{meV}\right.$ and $\left.\mathbf{Q}=(1,0,5.2)\right](\mathbf{I})$. 


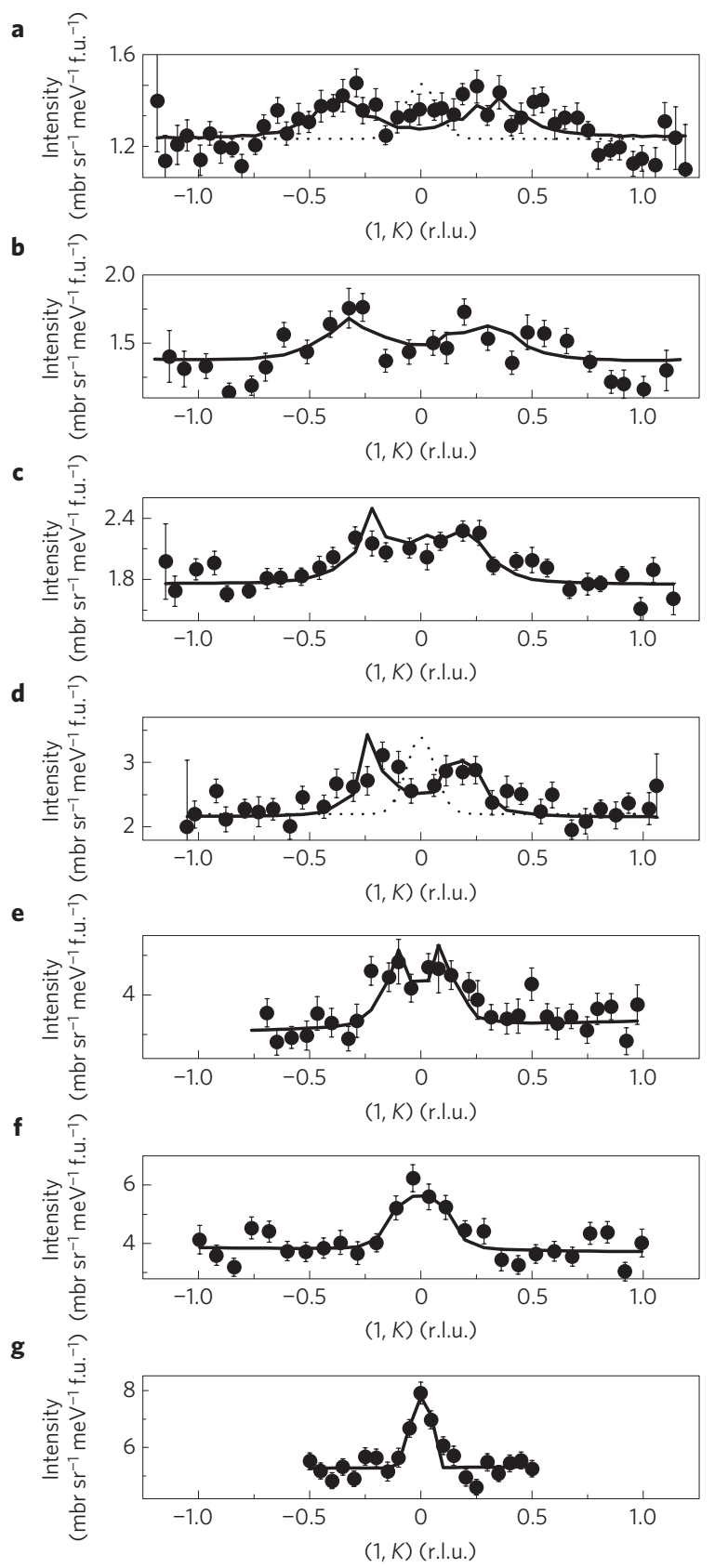

Figure 2 | Constant-energy cuts of the spin-wave dispersion as a function of increasing energy and our model fit using the Heisenberg

Hamiltonian. A series of constant-energy cuts through the antiferromagnetic spin-wave zone centre as a function of decreasing energy $E=144 \pm 20(\mathbf{a}) ; E=135 \pm 10(\mathbf{b}) ; E=115 \pm 15$ (c); $E=100 \pm 10$ (d); $E=64 \pm 10(\mathbf{e}) ; E=48 \pm 6(\mathbf{f}) ; E=25 \pm 5 \mathrm{meV}(\mathbf{g})$. The solid lines are model fits to the data after convoluting the cross-section to the instrumental resolution. Typical instrumental resolutions are shown as dotted lines in $\mathbf{a}$ and $\mathbf{d}$. Error bars indicate one sigma.

(Fig. 1h), $137 \pm 15$ (Fig. 1i), $135 \pm 10$ (Fig. 1j) and $144 \pm 15 \mathrm{meV}$ (Fig. 1k), counter-propagating spin-wave modes become apparent. The scattering changes from ring-like at $100 \mathrm{meV}$ (Fig. $1 \mathrm{~g}$ ) to ellipses elongated along the $K$-direction for energies above $110 \mathrm{meV}$ (Fig. 1h-k). For an energy transfer of $175 \pm 15 \mathrm{meV}$ (Fig. 11), spin waves show a broad square-like scattering already reaching the zone boundary in the $K$-direction.

To quantitatively determine the spin-wave dispersion, we cut through the two-dimensional images similar to Fig. 1 for various incident beam energies $\left(E_{\mathrm{i}}\right)$ aligned along the $c$ axis. Figure $2 \mathrm{a}-\mathrm{g}$ shows the outcome for different spin-wave energies in the form of constant- $E$ scans along the $K$-direction around the antiferromagnetic zone centre. As the excitation energy increases from $25 \mathrm{meV}$ (Fig. 2g) to $144 \mathrm{meV}$ (Fig. 2a), well-defined counterpropagating spin waves approach the zone boundary. To illustrate the general feature of the high-energy spin waves, we have used the scattering near $(2,0,0)$ r.l.u. as a background and assumed that the positive scattering at wave vectors below $(2,0,0)$ r.l.u. is entirely magnetic. Figure 3 a shows the outcome of the backgroundsubtracted scattering for the $E_{\mathrm{i}}=450 \mathrm{meV}$ data projected in the wave vector $(\mathbf{Q}=[1, K])$ and energy space. In spite of the spin-wave intensity modulation along the $L$-direction due to the exchange interaction $J_{c}$ between the FeAs planes ${ }^{23}$ (Fig. 1a), one can see three clear plumes of scattering arising from the in-plane antiferromagnetic zone centres $\mathbf{Q}=(1,-2),(1,0)$ and $(1,2)$ r.l.u. The spin-wave scattering disperses for energies above $100 \mathrm{meV}$ and extends up to about $200 \mathrm{meV}$. As spin waves become less dispersive as the zone boundary is approached, we locate the spin-wave excitations through energy scans at a fixed wave vector. Figure $3 c-h$ summarizes a series of such scans at different wave vectors that reveal clear dispersions near the zone boundary and a maximum spin-wave bandwidth of about $200 \mathrm{meV}$.

In addition to the results presented in Figs $1-3$, we have also collected similar data at other wave vectors throughout the Brillouin zone. The filled circles in Fig. 4a,b summarize our measured spinwave dispersions along the $[H, 0,1],[1,0, L]$ and $[1, K, 1]$ directions. To understand these data as well as the wave vector/energy $(\mathbf{Q}-E)$ dependence of the spin-wave intensities, we consider a Heisenberg Hamiltonian consisting of effective in-plane nearest-neighbours (Fig. $1 \mathrm{a}, J_{1 \mathrm{a}}$ and $J_{1 \mathrm{~b}}$ ), next-nearest-neighbour (Fig. $1 \mathrm{a}, J_{2}$ ) and outof-plane (Fig. 1a, $J_{\mathrm{c}}$ ) exchange interactions. The dispersion relations are given by $^{21-25}: E(q)=\sqrt{A_{q}^{2}-B_{q}^{2}}$, where

$$
\begin{gathered}
A_{q}=2 S\left[J_{1 \mathrm{~b}}(\cos (\pi K)-1)+J_{1 \mathrm{a}}+J_{\mathrm{c}}+2 J_{2}+J_{\mathrm{s}}\right] \\
B_{q}=2 S\left[J_{1 \mathrm{a}} \cos (\pi H)+2 J_{2} \cos (\pi H) \cos (\pi K)+J_{\mathrm{c}} \cos (\pi L)\right]
\end{gathered}
$$

$J_{s}$ is the single-ion anisotropy constant and $q$ is the reduced wave vector away from the antiferromagnetic zone centre. The neutron scattering cross-section can be written as $^{22}$ :

$$
\frac{\mathrm{d}^{2} \sigma}{\mathrm{d} \Omega \mathrm{d} E}=\frac{k_{\mathrm{f}}}{k_{\mathrm{i}}}\left(\frac{\gamma r_{0}}{2}\right)^{2} g^{2} f^{2}(Q) \mathrm{e}^{-2 W} \sum_{\alpha \beta}\left(\delta_{\alpha \beta}-Q_{\alpha} Q_{\beta}\right) S^{\alpha \beta}(Q, E)
$$

where $\left(\gamma r_{0} / 2\right)^{2}=72.65 \mathrm{mb} \mathrm{sr}^{-1}, g$ is the $g$-factor $(\approx 2), f(Q)$ is the magnetic form factor of iron $\mathrm{Fe}^{2+}, \mathrm{e}^{-2 W}$ is the Debye-Waller factor $(\approx 1$ at $10 \mathrm{~K}), Q_{\alpha}$ is the $\alpha$ component of a unit vector in the direction of $\mathbf{Q}, S^{\alpha \beta}(Q, E)$ is the response function that describes the $\alpha \beta$ spin-spin correlations and $k_{\mathrm{i}}$ and $k_{\mathrm{f}}$ are incident and final wave vectors, respectively. Assuming that only the transverse correlations contribute to the spin-wave cross-section and finite excitation lifetimes can be described by a damped simple harmonic oscillator with inverse lifetime $\Gamma$ (refs 28-30), we have

$$
S^{y y}(Q, E)=S^{z z}(Q, E)=S_{\text {eff }} \frac{\left(A_{q}-B_{q}\right)}{E_{0}\left(1-\mathrm{e}^{-E / k_{\mathrm{B}} T}\right)} \frac{4}{\pi} \frac{\Gamma E E_{0}}{\left(E^{2}-E_{0}^{2}\right)^{2}+4(\Gamma E)^{2}}
$$

where $k_{\mathrm{B}}$ is the Boltzmann constant, $E_{0}$ is the spin-wave energy and $S_{\text {eff }}$ is the effective spin. We analysed our data by keeping $S$ and $S_{\text {eff }}$ distinct following the practice of ref. 22.

We fitted the measured absolute intensity of spin-wave excitations and their dispersions in Figs $1-4$ by convoluting the above-discussed neutron scattering spin-wave cross-section with the instrument resolution using the Tobyfit program ${ }^{28-30}$. As 

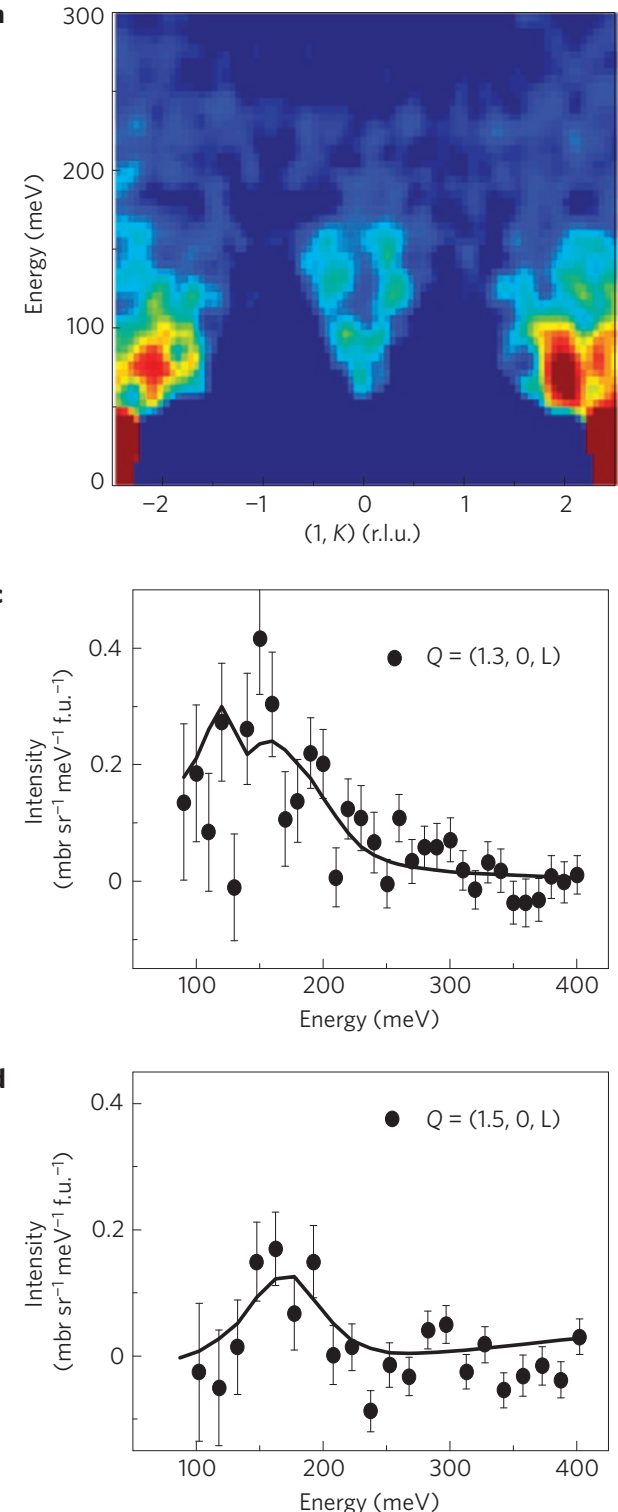

e

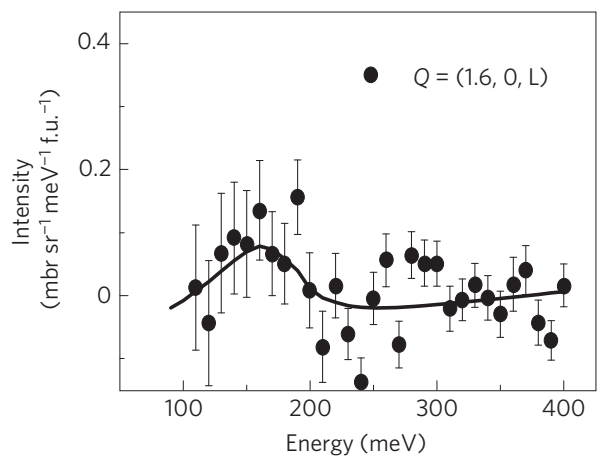

b

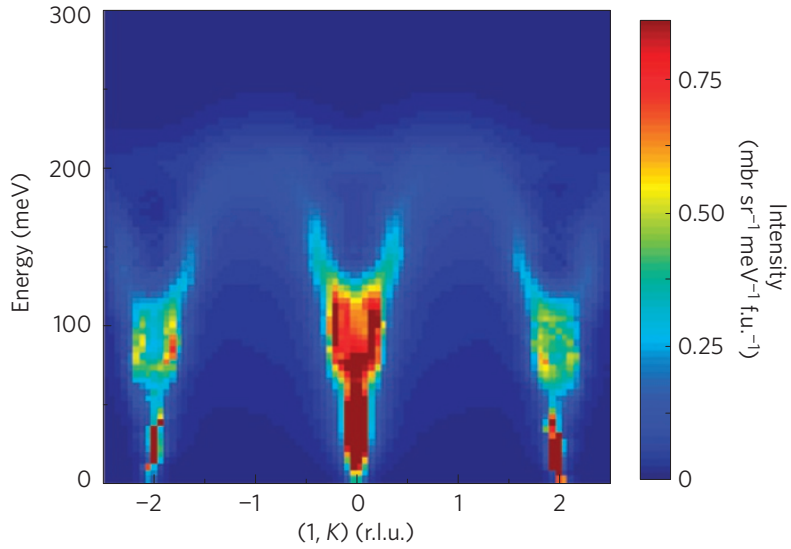

f

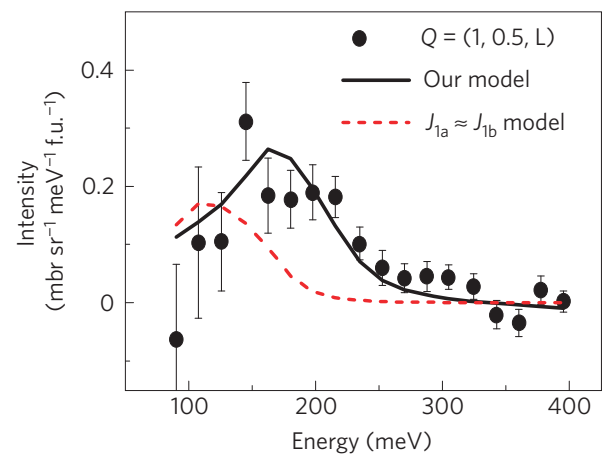

g

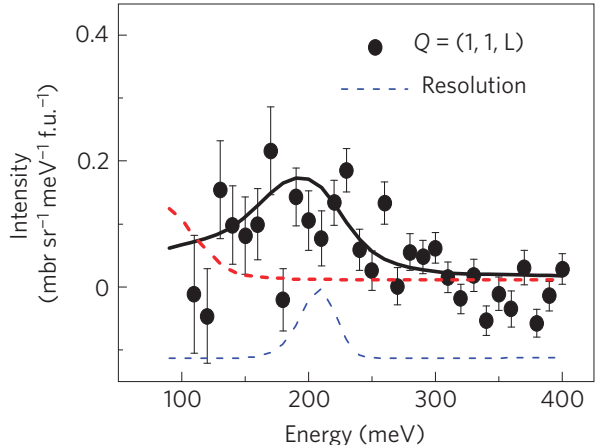

h

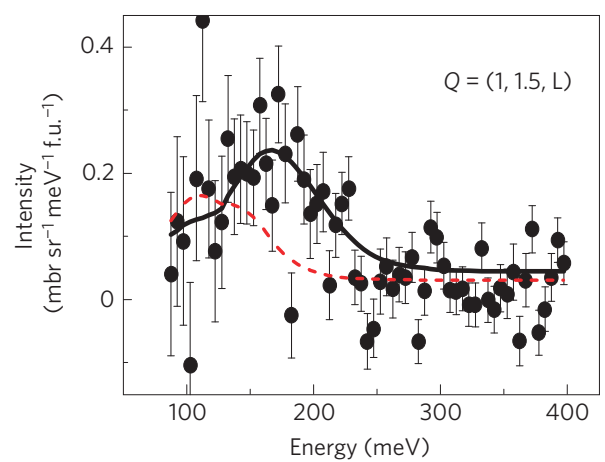

Figure 3 | Observed and calculated spin waves at $\mathbf{1 0} \mathbf{K}$, and constant-Q cuts near the antiferromagnetic zone boundary. a, The projections are in the scattering plane formed by the energy transfer axis and $(1, K)$ direction (with integration of $H$ from 0.8 to 1.2 r.l.u.) after subtracting the background integrated from $1.8<H<2.2$ and from $-0.25<K<0.25$. Data were obtained with $E_{\mathrm{i}}=450$ meV. $\mathbf{b}$, Calculated spin-wave excitations using the model

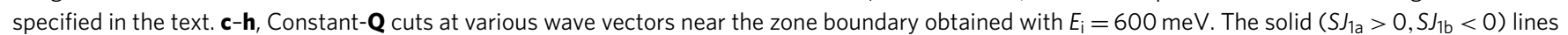
are our model fits to the data and the dashed lines are calculations assuming $S J_{1 a} \approx S J_{1 b}$. The error bars indicate one sigma.

$\mathrm{CaFe}_{2} \mathrm{As}_{2}$ exhibits tetragonal to orthorhombic lattice distortion below the $T_{\mathrm{N}}$ (ref. 27), care was taken to include the $(H, K) /(K, H)$ twin domains in the computed scattering cross-section. We find that the Heisenberg Hamiltonian with only the nearest-neighbours effective exchange couplings ( $J_{1 \mathrm{a}}$ and $J_{1 \mathrm{~b}}$ are finite, and $J_{2}=0$ ) cannot explain the data. Theoretically, it has been argued that the observed collinear spin structure in Fig. 1a is consistent with either $S J_{1 \mathrm{a}} \approx J_{1 \mathrm{~b}} \approx(1 / 2) S J_{2}$ or $S J_{1 \mathrm{a}} \approx 2 S J_{2} \gg S J_{1 \mathrm{~b}}$, and 

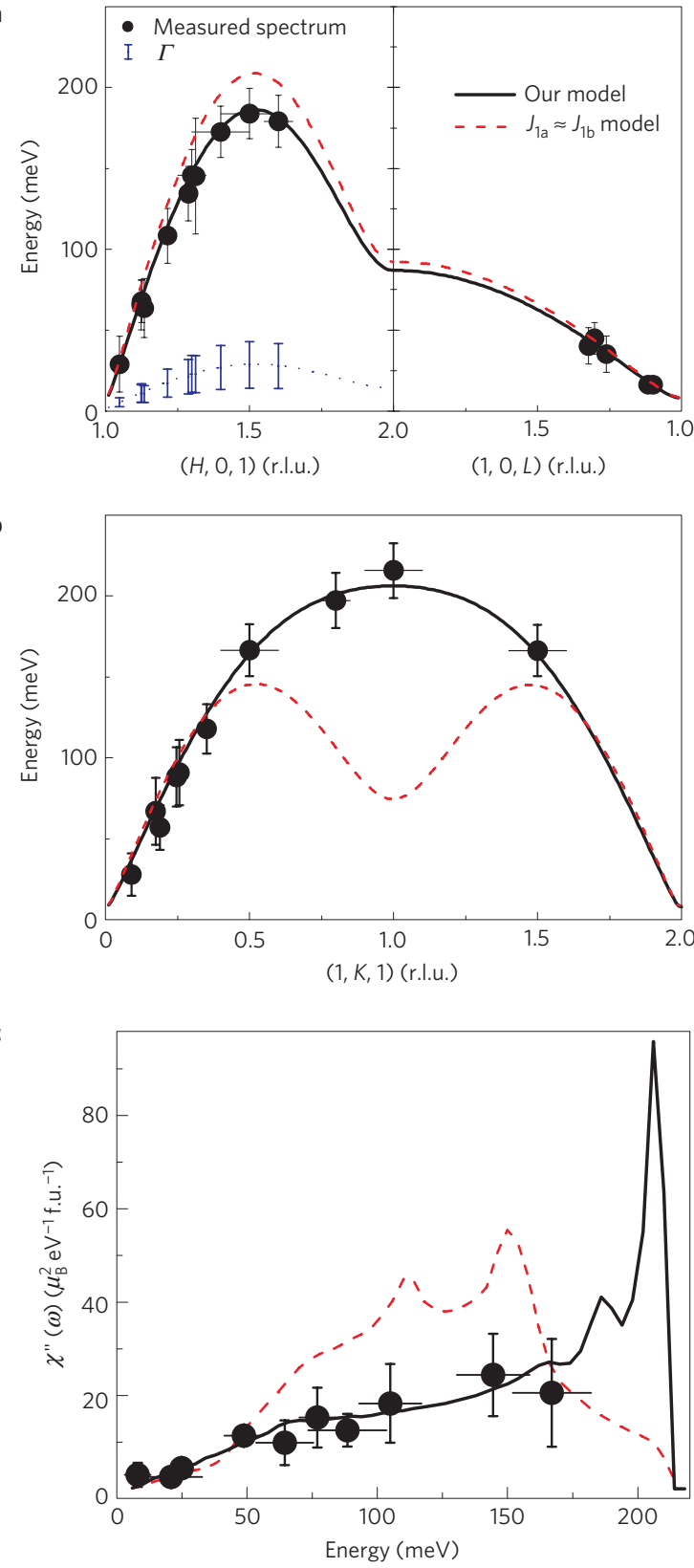

Figure 4 | Spin-wave dispersion relation along high-symmetry directions in the three-dimensional Brillouin zone and energy dependence of the local susceptibility. $\mathbf{a}, \mathbf{b}$, The filled circles are extracted from

constant- $E(-Q)$ cuts of various $E_{\mathrm{i}}$ data. The horizontal bars indicate the $E(Q)$ integration range and vertical bars are errors calculated from least-square fittings. Solid (dashed) lines are fits to the spin-wave models discussed in the text. The lengths of the blue vertical bars indicate the wave-vector dependence of $\Gamma$; the $\Gamma / E \sim 0.15$ is much smaller than that of metallic ferromagnet $\mathrm{La}_{2}-2 x \mathrm{Sr}_{1+2 x} \mathrm{Mn}_{2} \mathrm{O}_{7}$ where $\Gamma / E \sim 0.33-0.46$ (refs 28,29), thus suggesting a smaller influence of itinerant electrons in $\mathrm{CaFe}_{2} \mathrm{As}_{2}$. The blue dotted line is a guide to the eye. $\mathbf{c}$, Energy dependence of the local susceptibility ${ }^{2}$ obtained by integrating raw intensities above the background from $0.5<H<1.5 ;-0.5<K<0.5$, and $L$ from $L-0.5$ to $L+0.5$, where $L=1,3,5$ in the $(1,0, L)$ zone. The twinning effect has not been taken out. In our experimental set-up, the energy, magnetic form factor and polarization factors are all weakly $\mathbf{Q}$ dependent within the Brillouin zone. For simplicity, we used appropriate values for these factors at the zone centre $\mathbf{Q}=(1,0, L)$. Solid and dashed lines are the expected energy dependence of the local susceptibility for the two models discussed in the text with consideration of the twinning effect. distinguishing these two models requires spin-wave data near the zone boundary ${ }^{22}$.

The red dashed lines in Fig. $3 \mathrm{f}-\mathrm{h}$ show the expected zone-boundary spin waves assuming $S J_{1 \mathrm{a}}=27, S J_{1 \mathrm{~b}}=25, S J_{2}=36$ and $S J_{c}=5.3 \mathrm{meV}$. It is obvious that such a model failed to describe the zone-boundary data. Our best fits to both the low-energy and zone-boundary spin waves by independently varying the effective exchange parameters are shown as solid black lines in Figs 2 and 3 with $S J_{1 \mathrm{a}}=49.9 \pm 9.9, S J_{\mathrm{lb}}=-5.7 \pm 4.5, S J_{2}=18.9 \pm 3.4$ and $S J_{\mathrm{c}}=5.3 \pm 1.3 \mathrm{meV}$. The broadening of the spin waves with increasing energy is accounted for through $\Gamma \propto 0.15 E$ and shown as a blue dotted line in Fig. 4a. From our best fit to all spin-wave data, we find $S_{\text {eff }}=0.22 \pm 0.06$, which is smaller than previous measurements on powder samples of $\mathrm{BaFe}_{2} \mathrm{As}_{2}$ (ref. 22). The value of $S_{\text {eff }}$ and the measured $0.8 \mu_{\mathrm{B}} /$ Fe static moment ${ }^{27}$ suggest a $S \sim 1 / 2$ system.

From the fitting results in Figs 2-4, we see that the spin-wave dispersion and intensity in $\mathrm{CaFe}_{2} \mathrm{As}_{2}$ throughout the Brillouin zone can be well described by a Heisenberg Hamiltonian with effective nearest-neighbours and next-nearest-neighbour exchange interactions. Figure $4 \mathrm{a}, \mathrm{b}$ summarizes the spin-wave dispersions along all three high-symmetry directions and Fig. 4c shows the energy dependence of the local susceptibility ${ }^{7}$, together with calculations using $S J_{1 \mathrm{a}} \approx S J_{\mathrm{lb}}$ (red dashed lines) or our (solid lines) models. The former model clearly fails to describe the data. To test whether the spin-wave branch crosses the Stoner continuum as schematically illustrated in Fig. 1c, we plot spin-wave damping $\Gamma$ versus $E$ as a blue dotted line in Fig. 4a. Although $\Gamma$ is approximately proportional to $0.15 E$, there is no steep increase in $\Gamma$ at any wave vector indicative of a Stoner continuum (Fig. 1c). Instead, the observed spin-wave broadening at high energies may arise from magnon-electron scattering due to the low-temperature metallic nature of the system, similar to ferromagnetic metallic manganites ${ }^{28-30}$. Although these results may be consistent with $a b$ initio calculations presented in ref. 25, our data show welldefined spin waves near the zone boundary, in contrast to a simple picture of an electron-hole Stoner continuum extending to very high energies as in the case of pure metal $\mathrm{Cr}$ (refs 9, 10).

The central message of our work is that one can fit spin waves of $\mathrm{CaFe}_{2} \mathrm{As}_{2}$ throughout the Brillouin zone with a simple Heisenberg Hamiltonian without the need for a Stoner continuum-the hallmark of an itinerant electron system. In a spin-density-wave state driven by Fermi surface nesting of itinerant electrons, a Stoner continuum is expected to have an energy scale around $2 \Delta$, where $\Delta$ is the quasiparticle gap in the spin-density-wave state. Spin waves should be well-defined below $2 \Delta$, and quickly damp into a particlehole continuum above the characteristic energy. From Figs 1-4, we notice that there is no particular energy scale above which damped spin waves appear. This observation is in direct conflict with ref. 25, where a Stoner continuum is believed to set in above $100 \mathrm{meV}$. As our experiments were carried out on samples more than three times the mass and on an instrument with more neutron flux, the diminishing spin-wave scattering above $100 \mathrm{meV}$ in ref. 25 may simply arise from a poor signal-to-noise ratio of the measurement due to insufficient sample mass. The lack of direct evidence for a Stoner continuum below $200 \mathrm{meV}$ suggests weak low-energy electron-hole particle excitations. One local density approximation calculation has predicted essentially the correct in-plane magnetic exchange couplings $^{20}$; these results, however, are obtained within the tetragonal and collinear antiferromagnetic ordered structures contrary to the experiments. Furthermore, band-structure calculations suggest that the Fermi velocity $a / b$ anisotropy in $\mathrm{CaFe}_{2} \mathrm{As}_{2}$ is less than $8 \%$ in the low-temperature orthorhombic phase (D. J. Singh, personal communication). If spin-wave velocities in $\mathrm{CaFe}_{2} \mathrm{As}_{2}$ are proportional to $\left(v_{\mathrm{e}} v_{\mathrm{h}} / 3\right)^{1 / 2}$ such as those in chromium ${ }^{9}$, they should be similar along the $a / b$ directions. Although our results seem to favour a localized moment picture, a spin- $1 / 2$ model cannot 
be produced if all orbitals in iron are localized because there are even numbers of electrons per iron. Moreover, it is difficult to understand why direct and super-exchange interactions within the $\mathrm{Fe}-\mathrm{As}-\mathrm{Fe}$ plane are so different along the $a / b$ directions of the orthorhombic structure because the tetragonal to orthorhombic lattice distortion below $T_{\mathrm{N}}$ is small and only weakly affects the $\mathrm{Fe}-\mathrm{As}-\mathrm{Fe}$ bond distances/angles ${ }^{5,6}$. The observed large difference may hint at the involvement of other electronic degrees of freedom, such as orbital, in the magnetic transition. To achieve a comprehensive understanding of spin excitations, one must consider both the localized and itinerant electrons in these materials.

Received 11 March 2009; accepted 5 June 2009; published online 13 July 2009

\section{References}

1. Lee, P. A., Nagaosa, N. \& Wen, X.-G. Doping a Mott insulator: Physics of high-temperature superconductivity. Rev. Mod. Phys. 78, 17-85 (2006).

2. Kamihara, Y., Watanabe, T., Hirano, M. \& Hosono, H. Iron-based layered superconductor $\mathrm{La}\left[\mathrm{O}_{1-x} \mathrm{~F}_{x}\right] \mathrm{FeAs}(x=0.05-0.12)$ with $T_{\mathrm{c}}=26 \mathrm{~K}$. J. Am. Chem. Soc. 130, 3296-3297 (2008).

3. Chen, X. H. et al. Superconductivity at $43 \mathrm{~K}$ in $\mathrm{SmFeAsO}_{1-x} \mathrm{~F}_{x}$. Nature 453, 761-762 (2008).

4. Rotter, M., Tegel, M. \& Johrendt, D. Superconductivity at $38 \mathrm{~K}$ in the iron arsenide $\mathrm{Ba}_{1-x} \mathrm{~K}_{x} \mathrm{Fe}_{2} \mathrm{As}_{2}$. Phys. Rev. Lett. 101, 107006 (2008).

5. de la Cruz, C. et al. Magnetic order close to superconductivity in the iron-based layered $\mathrm{LaO}_{1-x} \mathrm{~F}_{x} \mathrm{FeAs}$ systems. Nature 453, 899-902 (2008).

6. Zhao, J. et al. Structural and magnetic phase diagram of $\mathrm{CeFeAsO}_{1-x} \mathrm{~F}_{x}$ and its relation to high-temperature superconductivity. Nature Mater. 7, 953-959 (2008).

7. Hayden, S. M. et al. Comparison of the high-frequency magnetic fluctuations in insulating and superconducting $\mathrm{La}_{2-x} \mathrm{Sr}_{x} \mathrm{CuO}_{4}$. Phys. Rev. Lett. 76 1344-1347 (1996).

8. Coldea, R. et al. Spin waves and electronic interactions in $\mathrm{La}_{2} \mathrm{CuO}_{4}$. Phys. Rev. Lett. 86, 5377-5380 (2001).

9. Fawcett, E. Spin-density-wave antiferromagnetism in chromium. Rev. Mod. Phys. 60, 209-283 (1998).

10. Endoh, Y. \& Böni, P. Magnetic excitations in metallic ferro- and antiferromagnets. J. Phys. Soc. Jpn 75, 111002 (2006).

11. Dai, J. H., Si, Q., Zhu, J. S. \& Abrahams, E. Iron pnictides as a new setting for quantum criticality. Proc. Natl Acad. Sci. USA 106, 4118-4121 (2009).

12. Fang, C., Yao, H., Tsai, W. F., Hu, J. P. \& Kivelson, S. A. Theory of electron nematic order in LaOFeAs. Phys. Rev. B 77, 224509 (2008).

13. Xu, C. K., Müller, M. \& Sachdev, S. Ising and spin orders in iron-based superconductors. Phys. Rev. B 78, 020501(R) (2008).

14. Ma, F., Lu, Z. Y. \& Xiang, T. Electronic structures of ternary iron arsenides $\mathrm{AFe}_{2} \mathrm{As}_{2}(\mathrm{~A}=\mathrm{Ba}, \mathrm{Ca}$, or Sr). Preprint at $<$ http://arxiv.org/abs/0806.3526> (2008).

15. Manousakis, E., Ren, J., Meng, S. \& Kaxiras, E. Is the nature of magnetic order in copper-oxides and in iron-pnictides different? Preprint at $<\mathrm{http}: / /$ arxiv.org/abs/0902.3450> (2009).

16. Dong, J. et al. Competing orders and spin-density-wave instability in $\mathrm{LaO}_{1-x} \mathrm{~F}_{x} \mathrm{FeAs}$. Eur. Phys. Lett. 83, 27006 (2008).
17. Yildirim, T. Frustrated magnetic interactions, giant magneto-elastic coupling, and magnetic phonons in iron-pnictides. Physica C 469, 425-441 (2009).

18. Mazin, I. I. \& Johannes, M. D. A key role for unusual spin dynamics in ferropnictides. Nature Phys. 5, 141-145 (2009).

19. Kariyado, T. \& Ogata, M. Normal state spin dynamics of five-band model for ion-pnictides. J. Phys. Soc. Jpn 78, 043708 (2009)

20. Han, M. J., Yin, Q., Pickett, W. E. \& Savrasov, S. Y. Anisotropy, itinerancy, and magnetic frustration in high $-T_{\mathrm{c}}$ iron pnictides. Phys. Rev. Lett. 102, 107003 (2009).

21. Zhao, J. et al. Low energy spin waves and magnetic interactions in $\mathrm{SrFe}_{2} \mathrm{As}_{2}$. Phys. Rev. Lett. 101, 167203 (2008).

22. Ewings, R. A. et al. High-energy spin excitations in $\mathrm{BaFe}_{2} \mathrm{As}_{2}$ observed by inelastic neutron scattering. Phys. Rev. B 78, 220501(R) (2008).

23. McQueeney, R. J. et al. Anisotropic three-dimensional magnetism in $\mathrm{CaFe}_{2} \mathrm{As}_{2}$. Phys. Rev. Lett. 101, 227205 (2008).

24. Matan, K., Morinaga, R., Iida, K. \& Sato, T. J. Anisotropic itinerant magnetism and spin fluctuations in $\mathrm{BaFe}_{2} \mathrm{As}_{2}$ : A neutron scattering study. Phys. Rev. B 79, 054526 (2009).

25. Diallo, S. O. et al. Itinerant magnetic excitations in antiferromagnetic $\mathrm{CaFe}_{2} \mathrm{As}_{2}$. Phys. Rev. Lett. 102, 187206 (2009).

26. Wu, G. et al. Different resistivity response to spin density wave and superconductivity at $20 \mathrm{~K}$ in $\mathrm{Ca}_{1-x} \mathrm{Na}_{x} \mathrm{Fe}_{2} \mathrm{As}_{2}$. J. Phys. Condens. Matter 20 , 422201 (2008).

27. Goldman, A. I. et al. Lattice and magnetic instabilities in $\mathrm{CaFe}_{2} \mathrm{As}_{2}$ : A single-crystal neutron diffraction study. Phys. Rev. B 78, 100506(R) (2008).

28. Perring, T. G. et al. Spectacular doping dependence of interlayer exchange and other results on spin waves in bilayer manganites. Phys. Rev. Lett. 87, 217201 (2001).

29. Perring, T. G. et al. <http://tobyfit.isis.rl.ac.uk/Main_Page>

30. Ye, F. et al. Spin waves throughout the Brillouin zone and magnetic exchange coupling in the ferromagnetic metallic manganites $\mathrm{La}_{1-x} \mathrm{Ca}_{x} \mathrm{MnO}_{3}(x=0.25$, 0.30). Phys. Rev. B 75, 144408 (2007).

\section{Acknowledgements}

We thank A. T. Boothroyd, T. Perring, D. Singh and A. Nevidomskyy for helpful discussions. This work is supported by the US National Science Foundation through DMR-0756568 and by the US Department of Energy, Division of Materials Science, Basic Energy Sciences, through DOE DE-FG02-05ER46202. This work is also supported in part by the US Department of Energy, Division of Scientific User Facilities, Basic Energy Sciences. The work at the Institute of Physics, Chinese Academy of Sciences, is supported by the Chinese Academy of Sciences. The work at USTC is supported by the Natural Science Foundation of China, the Chinese Academy of Sciences and the Ministry of Science and Technology of China.

\section{Author contributions}

P.D. and J.Z. planned the experiment. X.F.W., G.W. and X.H.C. fabricated the samples J.Z. and S.L. co-aligned the samples. J.Z., D.T.A., R.B. and P.D. carried out the neutron experiments and data analysis. D.-X.Y. and J.H. helped with data analysis.

P.D. and J.Z. wrote the paper with input from other coauthors.

\section{Additional information}

Reprints and permissions information is available online at http://npg.nature.com/ reprintsandpermissions. Correspondence and requests for materials should be addressed to P.D 